\title{
Filtration model of the unsteady suspension flow in a porous medium
}

\author{
Ludmila Kuzmina ${ }^{1}$, and Yuri Osipov ${ }^{2, *}$ \\ ${ }^{1}$ National Research University Higher School of Economics, 101000 Moscow, Russia \\ ${ }^{2}$ Moscow state university of civil engineering, Yaroslavskoye shosse, 26, Moscow, Russia, 129337
}

\begin{abstract}
The study of filtration of the suspension in a porous medium is a vital problem in the design and construction of tunnels and hydraulic structures. An exact solution is constructed for an unsteady flow of a monodisperse suspension in a homogeneous porous medium with sizeexclusion mechanism for particle retention. The concentrations of suspended and precipitated particles are calculated in case of a linear blocking filtration coefficient.
\end{abstract}

\section{Introduction}

The study of the motion of the tiny solid particles in a porous soil is a vital problem in the design and construction of tunnels and hydraulic structures. During the filtration of the suspension in a porous medium some of the suspended particles pass through the pores along with the flow of the carrier fluid and others get stuck in the filter pores. Deep bed filtration, accompanied by the formation of deposit in the entire porous medium, and not only in its surface layer, significantly affects the properties of rocks and soil layers $[1,2]$. In a variety of technical and biological systems for cleaning surface and waste water and liquid industrial waste, solid particles are retained when the suspension moves through the filter medium [3].

The mathematical model of the particle transport and retention in a filter is based on the mechanical and geometric interaction of particles with a porous medium [4-6]. A homogeneous porous medium with pores having the same cross section throughout its entire length is considered. It is assumed that the particles pass freely through large pores and get stuck at the entrance of pores if the particle diameter is larger than the pore diameter. One pore is blocked by one particle and vice versa. The retained particles can not be knocked out of the pores by the carrier fluid flow or another suspended particles, and remain permanently in the pores. As the retained particles concentration grows, the number of free small pores decreases, and the rate of deposit formation slows down. If all small pores are clogged by the retained particles, the retained particles concentration $S$ reaches the maximum possible limit value $S_{M}$. The coefficient of proportionality $\Lambda(S)$ between the growth rate of the deposit and the suspended particles concentration is called the

\footnotetext{
* Corresponding author: yuri-osipov@mail.ru
} 
filtration coefficient. The blocking filtration coefficient tends to zero when all small pores are blocked $\Lambda\left(S_{M}\right)=0$.

For different filtration models of a stationary suspension flow in a porous medium, exact and asymptotic solutions are obtained [7-12]. In the absence of analytical solutions, numerical calculations are performed [13-15].

Standard filtration models for the suspension flow in a porous medium assume that a suspension with constant suspended particles concentration is injected into the filter inlet. In the paper the exact solution of the filtration problem for a suspension with variable concentration of suspended particles at the filter inlet is obtained. Numerical calculations of the suspended and retained particles concentrations are performed for a linear blocking filtration coefficient.

\section{Governing equations}

Consider two basic dimensionless equations for the suspended and retained particles of a suspension in a porous medium. Let $C(x, t)$ is the concentration of suspended particles, $S(x, t)$ is the retained particles concentration. The particles concentrations satisfy the equation of continuity, which, taking into account the immobility of the deposit, has the form

$$
\frac{\partial C}{\partial t}+\frac{\partial C}{\partial x}+\frac{\partial S}{\partial t}=0
$$

The growth rate of the retained particles concentration is proportional to the concentration of suspended particles from which it is formed. It also depends on the current value of the retained particles concentration $S(x, t)$ : the more pores of small diameter are clogged by particles, the slower the growth rate of the deposit. The equation for deposit growth is

$$
\frac{\partial S}{\partial t}=\Lambda(S) C
$$

Here the filtration coefficient $\Lambda(S)>0$ for $S \geq 0$.

The system of equations (1), (2) is considered in the domain

$$
\Omega=\{0<x<1, t>0\} .
$$

The boundary conditions for the system (1), (2) are given at the filter inlet $x=0$ and at the initial time $t=0$ :

$$
\begin{gathered}
\left.C(x, t)\right|_{x=0}=p(t) ; \\
\left.C(x, t)\right|_{t=0}=0 ;\left.\quad S(x, t)\right|_{t=0}=0
\end{gathered}
$$

Conditions (4) indicate that at the initial moment $t=0$ the porous medium does not contain any suspended and retained particles. A suspension with variable suspended particles concentration (3) is injected into the porous medium and moves in the filter with the velocity $v=1$. The characteristic line $t=x$ of the equation (1) - the front of the suspended and retained particles concentrations is the mobile boundary of the suspension. 
In the subdomain of an empty porous medium $\Omega_{0}=\{0<x<1,0<t<x\}$ the solution is zero; in the subdomain of the suspension $\Omega_{S}=\{0<x<1, t>x\}$ the solution is positive.

\section{The solution at the filter inlet}

At the filter inlet $x=0$ the equation (2) has the form

$$
\frac{\partial S}{\partial t}=\Lambda(S) p(t)
$$

Division of both sides of (5) by $\Lambda(S)$ and integration with respect to the variable $t$ gives

$$
\int_{0}^{t} \frac{\partial S / \partial t}{\Lambda(S)} d t=P(t)
$$

where $P(t)=\int_{0}^{t} p(t) d t$.

Using condition (4), the integral in the left part of (6) can be written as

$$
\int_{0}^{S(0, t)} \frac{d S}{\Lambda(S)}=P(t) .
$$

Formula (7) specifies the retained particles concentration at the filter inlet.

\section{The solution on the concentrations front}

Denote the characteristic variables

$$
\tau=t-x, x=x
$$

Equation (1) takes the form

$$
\frac{\partial C}{\partial x}+\frac{\partial S}{\partial \tau}=0
$$

On the suspended and retained particles concentrations front $t=x$ the deposit concentration $S=0$. Substitution of (2) into (8) gives

$$
\frac{\partial C}{\partial x}+\Lambda(0) C=0
$$

The solution of the equation (9) with the condition $\left.C\right|_{x=0}=p(0)$ is

$$
C=p(0) e^{-\Lambda(0) x}
$$




\section{The exact solution of the filtration problem}

From (2)

$$
C=\frac{\partial S / \partial t}{\Lambda(S)}
$$

Substitution of (11) into (1) gives

$$
\frac{\partial}{\partial t}\left(\frac{\partial S / \partial t}{\Lambda(S)}\right)+\frac{\partial}{\partial x}\left(\frac{\partial S / \partial t}{\Lambda(S)}\right)+\frac{\partial S}{\partial t}=0 .
$$

Using the formula

$$
\frac{\partial}{\partial x}\left(\frac{\partial S / \partial t}{\Lambda(S)}\right)=\frac{\partial}{\partial t}\left(\frac{\partial S / \partial x}{\Lambda(S)}\right)
$$

the equation (12) can be transformed to

$$
\frac{\partial}{\partial t}\left(\frac{\partial S / \partial t}{\Lambda(S)}\right)+\frac{\partial}{\partial t}\left(\frac{\partial S / \partial x}{\Lambda(S)}\right)+\frac{\partial S}{\partial t}=0 .
$$

Integration of (13) with respect to the variable $t$ gives

$$
\frac{\partial S / \partial t}{\Lambda(S)}+\frac{\partial S / \partial x}{\Lambda(S)}+S=K(x)
$$

The function $K(x)$ is determined by the initial condition (4): $K(x)=0$.

The equation (14) in the characteristic variables takes the form

$$
\frac{\partial S / \partial x}{\Lambda(S)}+S=0
$$

Transfer of the term $S$ to the right side of (15), division by $S$, and integration with respect to $x$ gives

$$
\int_{0}^{x} \frac{\partial S / \partial x}{S \Lambda(S)} d x=-x
$$

After the change of the variable in the integral equation (16) takes the form

$$
\int_{S(0, \tau)}^{S(x, \tau)} \frac{d S}{S \Lambda(S)}=-x .
$$

An inverse change of variables gives

$$
\int_{S(0, t)}^{S(x, t-x)} \frac{d S}{S \Lambda(S)}=-x .
$$


Formulas (7), (11), (18) determine the exact solution of the problem (1) - (4) in the domain $\Omega_{S}$.

\section{The linear filtration coefficient}

Consider a model with the linear blocking filtration coefficient

$$
\Lambda(S)=\lambda\left(S_{M}-S\right), S_{M}>0 .
$$

In this case, the retained particles concentration at the filter inlet $x=0$

$$
S(0, t)=S_{M}\left(1-e^{-\lambda P(t)}\right)
$$

In the domain $\Omega_{S}$ the suspended and retained particles concentrations are given by explicit formulas

$$
S(x, t)=\frac{S_{M}\left(e^{\lambda P(t-x)}-1\right)}{e^{\lambda S_{M} x}+e^{\lambda P(t-x)}-1} ; \quad C(x, t)=\frac{e^{\lambda P(t-x)} p(t-x)}{e^{\lambda S_{M} x}+e^{\lambda P(t-x)}-1} .
$$

\section{Numerical calculation of the filtration periodic mode}

Let the suspended particles concentration at the filter inlet vary periodically: $p(t)=1-\cos \pi t$. The calculation of the filtration problem (1) - (4) with the filtration coefficient (19) was performed for the parameters $\lambda=1, S_{M}=1$.

Fig. 1 shows the graphs of the suspended particles concentration: a) the time dependence at the filter inlet $x=0$ and the filter outlet $x=1 ; \mathrm{b})$ the dependence on the coordinate $x$ for $t=1$ and $t=3$ (decreasing), $t=2$ and $t=4$ (increasing), and limit values for large even and odd integers $t$. The dashed line indicates the maximum value $C=2$ of the suspended particles concentration.

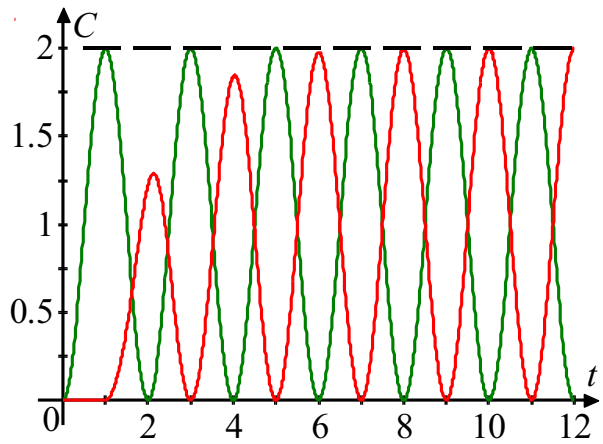

Fig. 1. a) The graphs of $\left.C(x, t)\right|_{x=0}$ and $\left.C(x, t)\right|_{x=1}$.

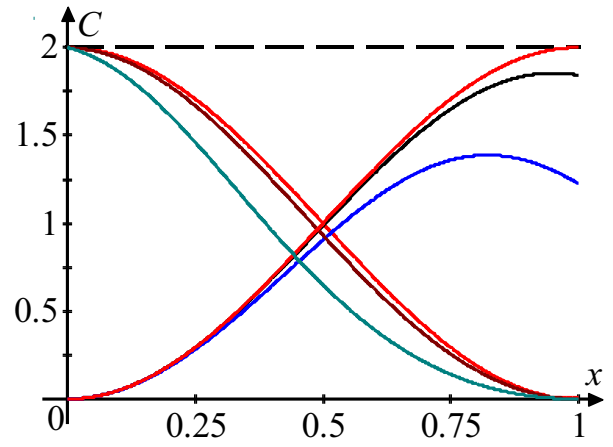

b) The graphs of $\left.C(x, t)\right|_{t=\text { const }}$.

Fig. 2 presents the graphs of the retained particles concentration: a) time dependence at the filter inlet (left) and filter outlet (right); b) dependence on the coordinate $x$ for $t=0.5,1,2,5$ (from bottom to top). The dashed line indicates the maximum limit value $S=1$ of the retained particles concentration. 


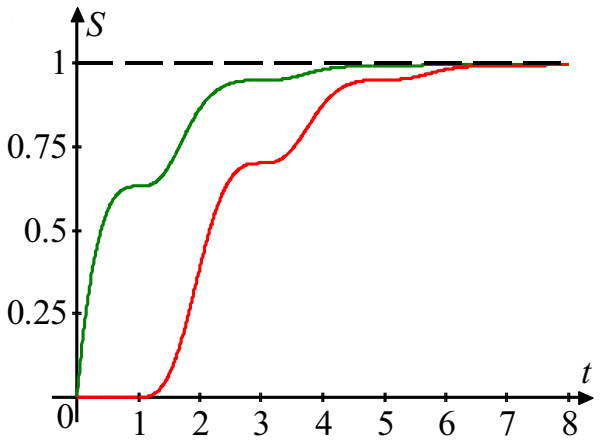

Fig. 2. a) The graphs of $\left.S(x, t)\right|_{x=0}$ and $\left.S(x, t)\right|_{x=1}$.

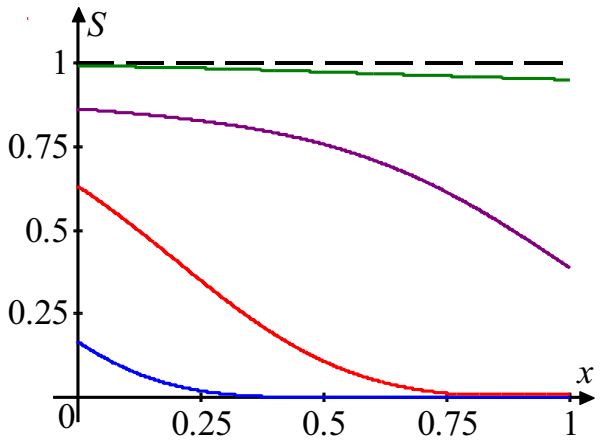

b) The graphs of $\left.S(x, t)\right|_{t=c o n s t}$.

\section{Conclusion}

The exact solution of the one-dimensional filtration problem of a suspension in a homogeneous porous medium for any variable concentration of suspended particles at the filter inlet is constructed. In the case of a linear blocking filtration coefficient, explicit analytic formulas are obtained for the solution, generalizing the known solution for the flux of a suspension of constant concentration [7].

According to Fig. 1 a), b) the suspended particles concentration quickly goes to the periodic mode with the period of the injected suspension concentration at the filter inlet. With increasing time, the retained particles concentration tends to the limiting value (Figures 2a) and b)), which is determined by the blocking filtration coefficient (19).

The exact solution gives way to simplify the determination of parameters of the filtration process in laboratory and field conditions [16].

\section{References}

1 P.G. Bedrikovetsky, Mathematical Theory of Oil and Gas Recovery (Kluwer 1994)

2 K.S. Basniev, N.M. Dmitriev, Mechanics of fluid flow (Wiley, 2012)

3 C. Noubactep, S. Caré, Chem. Eng. J. 163 (2010)

4 Z. You, A. Badalyan, P. Bedrikovetsky, SPE J. 18 (2013)

5 A. Badalyan, Z. You, K. Aji, P. Bedrikovetsky, T. Carageorgos, A. Zeinijahromi, Rev. Sci. Instrum 85, ID 015111 (2014)

6 F. Bashtani, S. Ayatollahi, A. Habibi, M. Masihi, J. Memb. Sci. 435 (2013)

7 J. P. Herzig, D. M. Leclerc, P. Legoff, Ind. Eng. Chem. 62 (1970)

8 E.A. Vyazmina, P.G. Bedrikovetskii, A.D. Polyanin, Theor. Found. Chem. Eng. 41, 5 (2007)

9 Z. You, P. Bedrikovetsky, L. Kuzmina, Abstr. Appl. Anal. 2013, ID 680693 (2013)

10 Z. You, Y. Osipov, P. Bedrikovetsky, L. Kuzmina, Chem. Eng. J. 258 (2014)

11 L.I. Kuzmina, Yu.V. Osipov, Procedia Eng. 153 (2016)

12 M. Hayek, Appl. Math. Model. 38 (2014).

13 N. Sefrioui, A. Ahmadi, A. Omari, H. Bertin, Coll. Surf. A 427 (2013)

14 Yu.P. Galaguz, G.L. Safina, Procedia Eng. 153 (2016)

15 Y. Galaguz, G. Safina, Matec Web Conf. 86, 03003 (2016)

16 P.G. Bedrikovetsky, D. Marchesin, F. Checaira, A.L. Serra, E. Resende, J. Petr. Sci. Eng. 323 (2001) 